\title{
Optimized inspection design for the thermographic characterization of sub-pixel sized through cracks
}

\author{
Andrew Loeb ${ }^{\mathrm{a}, *}$, Christopher Earls ${ }^{\mathrm{a}, \mathrm{b}}$ \\ ${ }^{a}$ Center for Applied Mathematics, Cornell University, Ithaca, NY 14850, United States \\ ${ }^{b}$ School of Civil and Environmental Engineering, Cornell University, Ithaca, NY 14850, United States
}

\begin{abstract}
Non-destructive evaluation of structural components is critical for reducing costs from unnecessary replacements and maintenance. We study the utility of a non-contact modality for the inspection of thin metal plates for the presence of through cracks. Sensitivity to early stages of deterioration allows for simpler and less expensive repair than if a flaw propagates and becomes more damaging. Hence, we focus on the characterization of very small cracks with a thermal imaging technique. Through cracks interact with the flow of heat within a component, so that the characterization of cracks from a thermal image amounts to solving an inverse problem to discover unknown parameters that describe the crack.

We consider cracks with length of less than a millimeter, falling under the pixel resolution of the recording thermal camera. Although these flaws are not directly visible from imaging data, the well-understood theory of heat conduction can be used in inference of crack properties. Herein we present a method to design an inspection modality that yields optimal data for such inference. Numerical experiments are performed to compare our optimized inspection setup to previous thermographic inspection scenarios found in the literature. Our design is found to produce the same quality of inference as these previous experiments which require much more expensive equipment (e.g. more powerful lasers and more sensitive IR cameras).
\end{abstract}

Keywords: Active thermography, Through cracks, Inverse problems, Inspection optimization

\section{Introduction}

The use of pulsed laser thermography for non-destructive evaluation has been incrementally developed over the past decade. The method has been shown to be viable for detecting through cracks in the thin aluminum panels that compose the outer skin of airframes, for example. Previous work in the literature has mostly considered the detection and characterization of cracks that are several millimeters in length [1-5]. Flaws of this size may

\footnotetext{
*Corresponding author

Email addresses: ael89@cornell.edu (Andrew Loeb), earls@cornell.edu (Christopher Earls)
} 
have already developed past the point of safe operation. In the current paper we focus on nascent-stage defects: cracks that are contained within the area measured by a single pixel of the infrared imaging system which is used in the inspection.

Cracks this small require a careful approach to characterize with currently-available tools. In particular, we aim to design an inspection modality that uses no more than an inexpensive infrared imaging system (e.g. 10,000 USD) and laser of modest power (e.g. 10 W). The specifications of the thermal camera we model are taken from an entry-level research instrument, and the laser power we consider in our simulations is consistent with previous laboratory experiments in the literature $[1-3,6]$. The development of the proposed inspection design methodology will be done using a rigorous consideration of the mathematical theory of heat conduction. Each choice in the setup and performance of the thermal measurement is made to provide optimal data for the characterization of a sub-pixel crack in light of the theory. With the general utility offered by the proposed framework for optimized inspection design, flaws can be detected and characterized more clearly, and at earlier stages of their formation. This will result in more effective strategies for safe and inexpensive maintenance across a range of material properties.

\subsection{Scope and Organization}

This paper is divided into five sections. In Section 2, the history of active thermography, as it has been developed for this class of problem, is summarized. In Section 3, the mathematical framework is described in terms of a partial differential equation and its solution. The hypothesis on which our method is founded is motivated and stated, and details about the practical solution to both the forward and inverse problems are described in that same section. Section 4 begins with the validation of our optimization hypothesis with numerical simulations. Then the optimization framework is carried out for characterizing sub-pixel cracks in thin metallic panels. The results of a stochastic inverse solution based on simulated data are presented in Section 4.4. Finally, Section 5 summarizes the conclusions of this paper and the significance of our method.

\section{Background and Motivation}

Infrared (IR) thermography is a nondestructive, noncontact evaluation technique characterized by using the thermal signal emitted from an object's surface in order to infer its internal structure [7]. There are two broad subclasses of thermography, founded on either a passive or an active thermal signal. In the case of active thermography, a heat source such as a flash lamp, continuous wave laser, or frequency modulated laser is used to impart heat energy into the specimen under evaluation. Characteristics of the heat source are known by the experimenter, as well as the salient thermal properties of the specimen. Then subsequent variations in the thermal response can be used to infer other unknown structural properties. A history of IR technology and the development of pulsed thermal NDT is presented in ref. [8].

Active themography is particularly well-suited for the detection of cracks penetrating through a thin, plate-like specimen. Preliminary studies demonstrated that, while cracks are 
among the most problematic types of defect for characterization with thermal NDT, they do block heat flow in the perpendicular direction [9-12]. This means that the heat from the known thermal input will be obstructed and produce a thermal response significantly different from a similar domain without a crack. Further studies that approached this problem with all three branches of modern scientific understanding (mathematical theory, experiments, and numerical simulation) are also available in the literature [1-5].

Chatterjee et al. provided a comparison of three laser-driven modalities - namely, pulsed, lock-in, and frequency modulated techniques [5]. It was concluded that the pulsed mode provided the best signal-to-noise ratio for defects that penetrate up to $1 \mathrm{~mm}$ into a structure. We adapt this latter mode in our proposed inspection design scheme because the thin panels we consider here have a total depth of only $1 \mathrm{~mm}$. Li et al. took steps to optimize the overall NDT process according to a mathematical model $[1,2]$. An "optimum" laser offset distance from a crack was presented for scanning pulse laser-line and laser-spot thermography, which was later used in experiments by others [3]. Finally, Burrows et al. performed experiments studying in-service testing conditions to determine the narrowest crack opening and lowest laser power required for crack detection with laser pulse thermography [4]. All of the foregoing studies showed promise when applied to specimens with defects of several millimeter length.

Other work has been done to characterize specific physical parameters of cracks that have been previously found or are assumed to exist. Schlichting et al. investigated the problem of determining the size of a surface crack that does not fully penetrate a thick structure with the precondition that its location was already known [3]. The depth and angle at which the crack penetrates the sample were then estimated through the use of pulsed laser thermography. More recently, Jeong et al. refined this problem to consider cracks of length less than a millimeter, fitting entirely within a single pixel of an IR image [6]. Earlier direct methods could not give suitable information regarding the characteristics of such small flaws. In later work, automated stochastic procedures were developed to solve the inverse problem associated with optically unresolvable cracks, and demonstrate the feasibility of an inspection method founded on such an approach [13].

We continue the foregoing line of inquiry $[6,13]$, aimed at the characterization of small cracks penetrating through plate-like components, as well as developing an optimization framework for designing a suitable inspection modality for a particular context. That is, we have determined guidelines for the setup of an active thermography inspection to yield optimally precise estimates of a crack's true physical characteristics. The inspection phase here is the second in a two-step process, as follows. First, the location of the crack must be somewhat known, e.g. Li et al. describe a second derivative method for locating defects from an IR image [2]. Alternatively, Bryan details a flexible and computationally fast method for detecting small cracks based on the reciprocity gap functional [14]. In a single pass, Bryan's method can detect many cracks in the presence of realistic noise and quantization error. While it does not reliably determine the crack length, the method is shown to provide a good estimate of the location and orientation of elongated sub-pixel cracks. With estimates of the locations of one or many cracks, we make a second pass over the sample, using the same equipment, so as to provide detailed information about these flaws. Ideally, the total 
inspection time should be brief, so it is better to not introduce new tools during this second pass: a condition that the present work satisfies. The subsequently gathered data can then be analyzed offline, to inform action that might be required in order to maintain or replace the fielded specimen. It is the second imaging pass which is the focus of the current work. We will henceforth also assume the scenario of a single narrow elliptical crack with a known orientation of the major axis, and with the location of its center, $\vec{x}_{c}$, known to within one millimeter. We seek to optimize the second pass inspection setup to fully characterize the crack. Our results will be robust under use with any investigation of small defects involving pulsed laser thermography and generalizable for any metallic material, based on its thermal properties.

\section{Problem Description}

The flow of heat within a solid medium is governed by the heat equation, a parabolic partial differential equation (PDE) [15]. In its full form, the heat equation models heat transfer over time, due to conduction, convection, and radiation within a given spatial domain. It may describe spatial and temporal addition of heat within the domain, or on its surfaces. Alternatively, these surfaces to be insulated and provide no heat transfer across them.

The current work considers a flat, $1 \mathrm{~mm}$ thick, $100 \mathrm{~mm} \times 100 \mathrm{~mm}$ aluminum 5052 panel. The relevant thermal properties for this material are its density, $\rho\left(2680 \mathrm{~kg} / \mathrm{m}^{3}\right)$, specific heat, $C(880 \mathrm{~J} / \mathrm{kg} \mathrm{K})$, and thermal conductivity, $k$ (assumed to be constant at $138 \mathrm{~W} / \mathrm{m}$ $\mathrm{K})$. The surfaces of the specimen, including the internal boundaries forming a crack, are assumed to be insulating.

Thermal energy provided from a laser beam is directed onto the front face of the panel. The laser is assumed to deposit thermal energy in the form of a Gaussian profile, centered at a point $\vec{x}_{l}[16]$

$$
f(\vec{x})=\frac{2 P}{\omega^{2}} \exp \left(-\frac{2\left|\vec{x}-\vec{x}_{l}\right|^{2}}{\omega^{2}}\right),
$$

where $P$ is the laser power and $\omega$ is the beam width (the radius at which the intensity has dropped to $1 / e^{2}$ of its peak value).

In addition to the assumptions stated previously, our mathematical model is simplified by assuming that the panel under inspection has perfect absorptivity (i.e. behaves as an ideal black body), so that all of the laser energy is converted to heat. We also neglect convection and radiation surface effects. These effects are found to be negligible over the short inspection times considered, as supported by analytical and numerical considerations, and in the literature [3]. Lastly, the domain is idealized as two-dimensional, doing away with the $1 \mathrm{~mm}$ thickness of the panel. This is realized in all following computations by multiplying the material density of aluminum by the neglected thickness, $h$ and setting the thermal diffusivity, $\kappa=k / \rho h C$. The $2 \mathrm{D}$ assumption is supported by the Biot number for our material and domain geometry being $1.8 \times 10^{-4}$, since any value less than 0.1 implies that temperature variation within the solid is negligible compared with boundary effects [17]. 
The foregoing simplification is further validated with comparison of $2 \mathrm{D}$ simulations with full 3D simulations that include all nonlinear heat transfer terms. The subsequent difference in the temperature responses between $2 \mathrm{D}$ and 3D models vanishes after passing through our model for the imaging system with finite spatial and temporal resolution, while computation time is vastly reduced. It is noted that our approach to solving an inverse problem for crack characterization requires many iterations of the simulated inspections, so these simplifying assumptions are a practical necessity.

The resulting PDE boundary value problem that describes this inspection scenario is

$$
\begin{cases}\frac{\partial T(\vec{x}, t)}{\partial t}-\kappa \nabla^{2} T(\vec{x}, t)=f(\vec{x}, t) / \rho h C & \text { in } \Omega \times(0, \infty), \\ -\frac{k}{h} \frac{\partial T(\vec{x}, t)}{\partial \vec{n}}=0 & \text { in } \Gamma_{\text {crack }} \cup \Gamma, \\ T(\vec{x}, t)=T_{0} & \text { on } \Omega \times\{t=0\},\end{cases}
$$

where $\vec{n}$ denotes the outward normal vector to the domain and $T_{0}$ is the initial, constant temperature of $25{ }^{\circ} \mathrm{C}$. The domain $\Omega$ is the $2 \mathrm{D}$ surface of the panel with boundary $\Gamma$ around the outside of the panel and along the edges of the crack, as depicted in Figure 1.

\subsection{Analytical Solution}

The PDE boundary value problem above has a closed-form solution in the case that the domain is infinitely wide and uncracked $\left(\Omega=\mathbb{R}^{2}\right)$. The analytic derivation of this solution is presented in Appendix A, along with a visualization of the time evolution of the general heat response.

The solution to Equation (1), but over the flawless, infinite domain is

$$
T(\vec{x}, t)=\frac{P}{4 \pi k}\left(\operatorname{Ei}\left(-\frac{2\left|\vec{x}-\vec{x}_{l}\right|^{2}}{\omega^{2}}\right)-\operatorname{Ei}\left(-\frac{2\left|\vec{x}-\vec{x}_{l}\right|^{2}}{\omega^{2}+8 \kappa t}\right)\right),
$$

where $\operatorname{Ei}(z)=-\int_{-z}^{\infty} \frac{\exp (-t)}{t} d t$, the exponential integral. Note that the solution is radially symmetric around $\vec{x}_{l}$, the laser peak. A cross-sectional view of the temperature response and its gradient are shown in Figure 2.

To obtain the analytical solution to Equation (1) on a finite, but flawless, and insulated domain, the method of images [15] is used with Equation (2). By superimposing a replica of Equation (2), as if a second laser were positioned symmetrically across the domain boundary (a mirror image), the temperature response is symmetric across the boundary. This results in no net heat transfer: equivalent to an insulated boundary condition. For the 2D problem, an infinite array of shifted images of the domain are necessary for an arbitrarily precise solution, as demonstrated in Figure 3. While the boundary effects are negligible for a laser peak further than $20 \mathrm{~mm}$ from an insulated edge, they are profound for a laser aimed near the boundary. We found convergence with error smaller than the sensitivity of our imaging system model for all laser positions on a $100 \mathrm{~mm} \times 100 \mathrm{~mm}$ domain. Nine images are required, which is feasible to implement computationally. This has all been further verified with comparisons to numerical simulations with the insulated boundary conditions imposed, using the same convergence criterion. Figure 4 compares this method with Equation (2) for varying laser peak positions. 


\subsection{Optimization Hypothesis}

One goal of the current work is to determine the optimal inspection setup for characterizing small through cracks using pulsed laser thermography. This type of flaw interrupts the flow of heat through a thin panel domain, due to the crack's insulating effect, or viewed another way, its interruption in the continuity of the conducting medium. Hence, all of the available information about the crack is contained in the disruption it causes in the thermal response: that is, the difference between the measured thermal response in a cracked specimen and the expected response from an uncracked domain. This information is subject to both instrument noise and quantization error from the thermal imaging charge-coupled device (CCD), consisting in our case of assumed microbolometers. Therefore, the inspection data are more useful if the thermal disruption caused by the crack is large in the sense that it exceeds the measurement noise floor. A simple way to measure the strength of the disruption signal is by finding the thermal gradient from one side of the crack to the other in the recorded temperature response. A steep change in temperature is a sign that information about the crack is well-interpretable above the noise floor. Figure 5 shows the thermal response of a cracked domain caused by an identical laser pulse at three different values for the distances from the crack to the laser peak, $\left|\vec{x}_{c}-\vec{x}_{l}\right|$. A distance that is neither too close, nor too far, results in a high thermal gradient. By seeking to maximize this gradient, either simulation or trial inspections can be employed to manually tune parameters of interest in the design of a particular inspection protocol. This idea of maximizing the thermal gradient across the crack has been used by Li, et al. to provide a coarse estimate for the "optimal" distance between the laser peak position and a crack that is several millimeters long [2]. Furthermore, the selection of the optimal heating duration for a different problem in thermal NDT is discussed by Marinetti et al. [18]. These works use experimental data and numerical modeling, respectively, in the selection of their chosen inspection parameters.

The analysis in Section 3.1 gives a powerful tool for mathematically designing an optimal inspection setup. Since we have a closed-form expression for the thermal response in a flawless domain, we can analytically evaluate how changes in any inspection design parameter (e.g. material properties, laser power, etc.) affect the solution. In particular, we may find the spatial gradient vector field of Equation (2) itself, and take its scalar magnitude in order to obtain a function to maximize with respect to any inspection design parameter, or set of parameters. While this is not necessarily equivalent to maximizing the thermal gradient across a crack during inspection, there are no equivalent analytical results for the response in such a domain. We reconcile this issue with the following hypothesis: An inspection design parameter is optimized for crack detection and characterization when Equation (2) has maximal gradient with respect to that parameter. This puts optimizing crack characterization in the framework of a constrained optimization problem that is not too difficult to solve. The validation of our hypothesis will be explored in Section 4.1.

\subsection{Forward Modeling}

The characterization of small cracks from thermal data can be posed as an inverse problem. One class of inverse problems are problems whose solution yields information about underlying parameters instantiating a system, leveraged against observations of that 
system's behavior. This is in contrast to a forward problem, which is the prediction of a system's behavior, based on its known physical properties and parameters. In our case, the forward problem is characterized by finding the thermal response of a flawed metal panel for a crack with known size, shape, and location. This can be solved using the heat equation and the finite element (FE) method, as a means of discretizing and numerically treating Equation (1).

In this work, we solve the forward problem as a weak form [15] using the FEniCS open source Python application programming interface (API) containing software classes that support the FE method [19]. The FEniCS API includes constructive solid geometry classes, with which it is straightforward to define a $2 \mathrm{D}$ domain with a crack of varying size and position. Using FEniCS, the heat equation PDE may then be solved over a graded, unstructured linear triangle FE mesh, which has a varying spatial resolution that is finer near the crack. A spatio-temporal convergence study is carried out to find the appropriate spatial mesh refinement, as well as needed time step size. Convergence is confirmed across successive refinements as well as with comparison to the analytical solution and a full 3D simulation, using the criterion described above when considering discretization used in modeling the imaging system. Convergence is observed for time steps of 0.02 seconds and a spatial mesh of first-order Lagrangian triangles comprising approximately 33,000 nodes. The exact number of nodes depends on the size of the crack, as the mesh is generated after defining the geometry of the domain.

The finite element model furnishes an approximation to the heat response on a continuum. From this, realistic surrogate experimental data are generated, as if they were recorded by an actual thermal imaging system, through the following process. Field variable output from the FE mesh unstructured nodes are interpolated onto a rectangular grid, then integrated over the area which would be captured by each pixel of the hypothetical imaging system. Next, independent, identically distributed (i.i.d.) Gaussian measurement noise is added to each pixel reading, in a manner that is consistent with the noise-equivalent temperature difference (NETD) of our assumed microbolometer system. Finally, the data are rounded to be consistent with specified resolution, $\Delta T$, that is associated with the quantization assumed in our image capture. We assume an entry level research camera (A325sc from FLIR Systems, Inc) as the basis for our modeled imaging system, which has a NETD of 50 $\mathrm{mK}$, a standard temperature range from $0{ }^{\circ} \mathrm{C}$ to $350{ }^{\circ} \mathrm{C}$, and 14 -bit data representation, for a thermal resolution of $\Delta T=0.02{ }^{\circ} \mathrm{C}$. The spatial resolution $(320 \times 240)$ and angle of view of this camera $\left(6^{\circ}\right)$ are determined to give a pixel size of $0.9 \mathrm{~mm} \times 0.9 \mathrm{~mm}$ with a standoff distance between the camera and the sample being tested of $2.7 \mathrm{~m}$.

\subsection{Inverse Problem Formulation}

In this work, we use the Markov chain Monte Carlo (MCMC) method in solving the inverse problem of crack characterization [20]. This is a particular approach to solving inverse problems, but other methods exist. For a detailed explanation of the MCMC approach, as it applied to thermal imaging (e.g. crack position and size), see Appendix B. The advantage of MCMC is that it furnishes an inverse solution in the form of a probability density function (PDF), called the posterior, which reflects the relative probability for values of unknown 
parameters based on the considerations of both measured data and their measurement uncertainty (encoded into the likelihood PDF) along with prior beliefs regarding the model parameters (encoded in the prior PDF). Several statistics of interest can then be computed from these posteriors. The posterior mean and variance are particularly useful, since our focus here is optimizing the preceding inspection setup to provide informative data. We use the variance of a posterior distribution as a measure of precision within the data. An inspection design that results in a tight distribution of values (i.e. small variance) for a parameter is preferred over one giving a wide distribution. We may then compare credible intervals within the solution, using different inspection design parameters. That is, inspection parameters that yield high "confidence" in the inverse problem solution are favorable over those that succumb to noise, and fail to provide clear signals regarding the inversion parameters of interest.

\subsection{Practical Considerations}

We make a number of simplifying assumptions in the mathematical formulation of the crack characterization problem. Their justifications and limitations are enumerated here. First, it has been stated that this study is particularly concerned with through cracks in plate-like components, where the crack lengths are smaller than the imaging resolution. Although this assumption presents the primary challenge of the study, it is useful for our solution method. This is because our optimization hypothesis relies on finding specific values of geometric parameters that maximize the gradient of an analytic function. In particular, the crack location is described as a single point, $\vec{x}_{c}$, regardless of its actual size or shape. As a result, small flaws are better-represented in the proposed framework than larger cracks that may be easier to detect outright, in the first place. Another simplifying assumption adopted for the flawed panel is its absorbtivity of laser energy: we assume full absorbtivity, but this can be effectively relaxed, changing only the laser power coefficient to reflect the decreased energy that is transmitted into the panel.

Next, there are practical limitations on the parameterization of the laser that is used as the thermal energy source. A more powerful laser will produce higher contrast in a thermal image, but this cannot be relied on as the only means of detection enhancement for two important reasons. In addition to high cost and increased risk of personal injury, if a given laser is too powerful, it poses a risk to the sample under inspection. Earls [13] discusses sensitization, which may occur in aluminum 5052 above $260^{\circ} \mathrm{C}$. To avoid damage to the specimen we are attempting to inspect, and to demonstrate that our proposed method can be used without prohibitively expensive hardware, we consider a $10 \mathrm{~W}$ laser. The other parameter of an ideal Gaussian laser source is the spot size. Optical lenses can be used to manipulate the spot size of a laser with knowledge of the distance between the laser source and the target. To focus this spot size to be very small, the sensitivity to this distance becomes dramatic. A relatively high divergence angle must be used, so that small changes in the standoff distance result in large relative changes in spot size. For this reason, small spot sizes are not favorable from a practical perspective. Finally, it is noted that the Gaussian profile of laser energy, rather than, say, uniform over the spot size, is a well-supported assumption [21]. 
Lastly, we comment on the effect of quantization error resulting from the thermal camera data representation precision. Although i.i.d. Gaussian measurement error due to electronic sensor noise is captured in our model, and drives the stochasticity of the inverse problem solution, through the form of the likelihood PDF, the quantization error due to the bit depth of the A/D converter in our assumed CCD is not included directly in our modeled imaging system. Since we rely on sensitivity to slight changes in a temperature response, low thermal resolution is as deleterious to our aims as are contamination from electronic sensor noise. On the other hand, higher temperatures, and in particular the resulting larger gradients will reduce the relative importance of such quantization error. Thus the highgradient optimization we present will overcome both forms of noise simultaneously. We investigate the tradeoffs between these two sources of error for varying severity, illustrated in Figure 6. For values of NETD/ $\Delta \mathrm{T}$ greater than 2, measurement noise is determined to dominate. This is the value at which the discretized normal distribution has a standard deviation within $1 \%$ of a smooth Gaussian. The camera we simulate here has parameters $\mathrm{NETD}=0.05{ }^{\circ} \mathrm{C}$ and $\Delta \mathrm{T}=0.02{ }^{\circ} \mathrm{C}$, giving a ratio of 2.5 . Thus quantization error may be neglected here.

\section{Results and Discussion}

\subsection{Validation of Optimization Hypothesis}

Before exploring the utility of the proposed optimized crack characterization inspection design, we first determine the validity of the hypothesis stated previously in Section 3.2. Towards this end, it is important to observe the relationship between the gradient magnitude of the analytic solution and the quality of inference in the associated inverse problem, prior to using this metric to optimize the inspection design. To do this, we isolate a single parameter, the distance between the crack and laser position, measured perpendicular to the crack major axis (the $x$ direction), and perform several surrogate experimental MCMC runs. IR imaging system sensitivity is neglected in these trials, so that the only sources of randomness involved in creating estimates of posterior credible intervals is in the selection of candidate steps in the chains and measurement noise in the initial data. Seven laser offset distances are chosen, based on the gradient of the analytical solution as a function of this distance. These include the maximizing location and points nearer and further from the crack, including two locations having the same gradient magnitude. Five MCMC inverse solutions are performed for each of these locations, with the same five sets of noise in the initial data. The variances of the resulting posterior distributions are shown in Figure 7.

We find monotonic behavior in the variance of the estimation, decreasing with increasing gradient at the analytical solution evaluated at each laser offset. Furthermore, trials for offsets yielding the same gradient result in variances that are nearly identical, despite coming from laser positions both nearer and further than the maximizing position. These results support the optimization hypothesis we use, and suggest a deep connection governing this observed relationship between estimate confidence and the gradient of the associated analytical solution. We proceed with an analysis optimizing the crack characterization inspection 


\begin{tabular}{|c|c|c|}
\hline Parameter & Optimal Trend & Value Used \\
\hline Laser offset & Depends on other parameters & $1.56 \mathrm{~mm}$ \\
\hline Pulse duration & Increasing (asymptotic) & $0.5 \mathrm{~s}$ \\
\hline Laser power & Increasing & $10 \mathrm{~W}$ \\
\hline Beam width & Decreasing (asymptotic) & $1 \mathrm{~mm}$ \\
\hline Material conductivity & Decreasing (asymptotic) & $138 \mathrm{~W} / \mathrm{m} \mathrm{K}$ \\
\hline Material density & Decreasing (asymptotic) & $2680 \mathrm{~kg} / \mathrm{m}^{3}$ \\
\hline Material specific heat & Decreasing (asymptotic) & $880 \mathrm{~J} / \mathrm{kg} \mathrm{K}$ \\
\hline
\end{tabular}

Table 1: Optimal trends for parameters in the crack characterization problem.

design, referring to optimal parameters as those which analytically produce maximal gradient.

\subsection{Optimal Trends for Parameters}

There are several modeling parameters in the mathematical formulation of the proposed laser pulse inspection modality. Some of these parameters can be controlled in an inspection, while others are properties of the material itself, or may be inherently fixed due to material selection. We report the optimal trends for all of the parameters in Table 1 . These are found by numerically maximizing the gradient magnitude of Equation (2) with respect to each parameter individually. Some parameters increase the gradient asymptotically, giving small marginal increases as the parameter is increased. These are labeled "asymptotic" in Table 1.

The particular values which we use in the simulation of this inspection are also tabulated. These are chosen with consideration of the optimal trends, keeping with realistic limitations. For example, the use of a more powerful laser would provide a clearer image, but quickly becomes cost-prohibitive and dangerous to use. Furthermore, parameters which asymptotically increase the thermal gradient are balanced between these dimishing gains and practical limitations. The behavior of the magnitude of the gradient function, as it depends on the pulse duration, is shown in Figure 8. A pulse duration of 0.5 seconds achieves $97 \%$ of asymptotic limit. The duration must be doubled to give another $1 \%$ increase towards the limit, so 0.5 seconds is chosen as the pulse duration for our idealized inspection design. The beam width is fixed at $1 \mathrm{~mm}$ for the reasons stated in Section 3.5, though we note that a tighter beam would offer some minor improvements in the inspection effectiveness. The angle made between the laser spot and the major axis of the crack is not included in the mathematical formulation. However, simulations have confirmed the intuition that the crack is most-easily detected when it is perpendicular to the flow of heat, a condition that is achievable using data from the first pass, as described in Section 2. Lastly, all of the modeling parameters specific to a given material are set according to values found in the literature for $\mathrm{Al} 5052$.

The distance between the laser spot and the center of the crack has the most interesting behavior. The optimal offset of the laser was stated by Li et al. to be one laser radius [2]. We found a nonlinear relationship for optimal location, based on all of the other parameters. The experiments and numerical test in ref. [2] are done with few data points, linear interpolation, 
and their selection is done based on the best single result. With the method used here, any particular specimen, material, and/or laser can be treated. Using the parameter set specified above for our proposed inspection design, the analytical gradient is $14 \%$ higher than if a laser offset of $1 \mathrm{~mm}$ was used (i.e. if the criterion of Li et al. had been applied).

\subsection{Optimal Laser Position for a Bounded Domain}

The analytical solution of Equation (2) over an infinite planar domain can be used to furnish a solution to the laser-heating problem over an uncracked bounded domain using the method of images described in Section 3.1. By considering a tessalation of Equation (2) centered at points symmetric over the domain boundary, there can be no net heat transfer along the line of symmetry, so as to satisfy the insulation condition specified in our problem description (Equation (1)). The effect of an insulated bounded domain is that heat accumulates on the boundary, causing an asymmetric thermal response, meaning: results display a dependency for the optimal laser offset based on the direction to the crack, as well as a bias to some directions (the gradient also loses its radial symmetry). Thus for a given crack location, we seek the optimal laser position within the domain, rather than simply the distance between the crack and the laser peak. We explore this new problem defined on our $100 \mathrm{~mm} \times 100 \mathrm{~mm}$ domain, independently considering 361 flaw locations uniformly spaced over the panel. For each, the laser peak position that maximizes the gradient magnitude of the approximated solution at that flaw location is found. The findings are summarized in Figure 9 .

A pattern emerges from these results near the boundary, but further away from it, the insulating effect is lost, and all radial positions around the flaw are equivalent in term of optimal gradient (represented by a circle having the optimal laser offset as its radius). It seems for this scenario, the existence of a boundary in the domain has no effect on optimization analysis for cracks more than $20 \mathrm{~mm}$ away. This observation should extend to any sort of inhomogeneity within the test domain. Hence, other flaws, rivets, or actual panel boundaries, which are not perfectly insulated in practice, do not affect the validity of our optimization results, unless the flaw under study is particularly near them. In this case, optimal inspection parameters can still be determined numerically, provided that the forward model describes the effects of these local features.

\subsection{MCMC-Based Inversion Results Using Optimal Experimental Conditions}

Two sets of Markov chain Monte Carlo samplings are performed for a specific crack characterization problem. An elliptical crack with a semi-major axis of $0.25 \mathrm{~mm}$ and semiminor axis of $0.03 \mathrm{~mm}$ is set in the center of a $100 \mathrm{~mm} \times 100 \mathrm{~mm}$ aluminum 5052 panel. This is smaller than any crack that has previously been studied in the literature on thermographic imaging. Lasers with spot size of $1 \mathrm{~mm}$ and heating times of $0.5 \mathrm{~s}$ are used in all samplings. Furthermore, the same thermal camera with pixel size, NETD, and thermal resolution stated above is considered. In the first set of 20 samplings (set 1), a laser power of $100 \mathrm{~W}$ is used, with $10 \mathrm{~mm}$ offset between the laser peak and the crack. This is done for comparison with reference to [13], as a benchmark representing previous related work from the literature. In the second set of simulations (set 2), the optimal distance between the laser and the 


\begin{tabular}{|c|c|c|c|c|c|}
\hline Crack Parameter & True Value & Set 1 Mean & Set 1 St. Dev & Set 2 Mean & Set 2 St. Dev \\
\hline$x$ location $(\mathrm{mm})$ & 0.0 & 0.05 & 0.12 & -0.01 & 0.14 \\
\hline$y$ location $(\mathrm{mm})$ & 0.0 & 0.02 & 0.24 & 0.02 & 0.20 \\
\hline Semi-major axis $(\mathrm{mm})$ & 0.25 & 0.25 & 0.03 & 0.24 & 0.03 \\
\hline
\end{tabular}

Table 2: Aggregated mean and variance from two sets of 20 MCMC samplings.

crack, $1.57 \mathrm{~mm}$, is used, and the laser power is set to only $10 \mathrm{~W}$. This laser power is chosen to be similar to what was used in prior experiments with larger flaws as a reasonable-cost instrument $[1-3,6]$. Additionally, the analytical gradient of the two sets of laser parameters are found to be close, 4.9 and 5.0, respectively, so they are expected to give similar confidence in their posterior estimation.

All posterior estimates here are gathered from MCMC sampling having 5,000 discarded burn-in steps and 20,000 saved samples. A separate study was done with a total of 300,000 samples, to affirm that these MCMC samplings are sufficiently long to believe that they are sampling from the actual stationary posterior probability densities of the various crack parameters. The methodology and results of this convergence study are detailed in Appendix C. The two simulation contexts have the same remaining underlying modeling parameters, each contaminated with i.i.d. Gaussian additive noise on the "true" data. These data are gathered from a separate finite element PDE solutions. Furthermore, each of the simulations are initialized at random values of the crack parameters of interest, taken from the support of the prior distribution.

We summarize the results of these MCMC samplings in three ways. First, the posterior statistics of the two sets of chains are directly compared. The mean and variance of each chain of 20,000 samples are aggregated, averaged over each sampling in the set, in Table 2. As we expect from the gradient of Equation 2 for each set, the two give nearly identical results. The standard deviations of each parameter estimate give insight into their relative importance. Since all three parameters are inferred from the same data, we see that the crack length has the strongest effect on the disruption of heat flow. After that, the location of the crack closer or further to the laser ( $x$ direction) is more important than its location in the perpendicular direction ( $y$ direction).

The form of the posterior distributions for the estimated parameters are also interesting. A strength of the MCMC method is that the solutions it provides for inverse problems have more information than just the statistics in Table 2. Since the chains sample from a probability distribution, the relative frequency of each value represents the probability that the noisy data were produced from that underlying model parameter value. Because of this, we are able to plot the histograms representing the estimated marginal distributions of each crack parameter in Figure 10. These are representative of the 40 total chains.

Finally, we present 100,000 total samples aggregated from ten independent MCMC samplings having the same additive noise and different random starting values (gathered for the convergence study in Appendix C). These samples are plotted in Figure 11 to show the relationship between the three parameters. Notable correlation is only seen between the 
crack length and the laser offset distance perpendicular to the major axis of the crack. This is because a small crack close to the laser spot will cause a disruption in the flow of heat similar to a larger crack further away.

\section{Conclusions}

In this paper we set out a method for designing optimal non-contact thermographic inspection approaches for characterizing small through cracks in thin metal panels. The method relies on a two-pass active thermography inspection modality employing a laser and a thermal camera. After potential flaws have been located with a preliminary pass of the thermographic equipment, a brief second inspection is done for each crack. The specific setup of this second inspection requires choices for several design parameters. We have developed and presented a mathematical framework for describing the optimal choice of these parameters, so that each crack is as clearly revealed as possible within the resulting thermal data. This framework is validated using finite element simulations and tested in comparison with previous experiments in which the values of these parameters were chosen in other ways. It is shown that a $10 \mathrm{~W}$ laser directed at the location that is found to be optimal in our method provides data that are equivalent to earlier experiments in which a $100 \mathrm{~W}$ laser was required.

Although the specific problem of small through cracks in thin aluminum panels is considered here, the framework for inspection design optimization that we describe is applicable for any metallic material. Furthermore, the particular MCMC solution to the inverse problem can be substituted by any other inverse solution method based on a user's time and accuracy constraints. The design parameters that our method produces are chosen to give data containing the most information about the underlying structure of the sample under evaluation.

\section{Acknowledgments}

This work was supported by the National Science Foundation (DGE-1144153).

\section{Appendix A. PDE Solution}

The heat equation PDE with Gaussian laser pulse heating has been previously studied in the literature. Lax presented the time-independent case of the temperature rise induced by a laser beam in a light-emitting semiconductor in 1977 [22]. Li et al. gave a similar timedependent derivation for the case of an elliptical Gaussian beam heating a 3D structure [1]. The derivation below will be independent of these earlier references, as discussion is limited to the specific case of a circular Gaussian beam heating an infinite 2D plane.

Using the notation from Section 3, the non-homogeneous boundary value problem is formulated as

$$
\left\{\begin{array}{ll}
\frac{\partial T(\vec{x}, t)}{\partial t}-\kappa \nabla^{2} T(\vec{x}, t)=f(\vec{x}, t) / \rho h C & \text { in } \mathbb{R}^{2} \times(0, \infty) \\
T(\vec{x}, t)=T_{0} & \text { on } \mathbb{R}^{2} \times\{t=0\}
\end{array},\right.
$$


with

$$
f(\vec{x}, t)=\frac{2 P}{\omega^{2}} \exp \left(-\frac{2\left|\vec{x}-\vec{x}_{l}\right|^{2}}{\omega^{2}}\right) .
$$

The Green's function for this PDE is

$$
\Phi(\vec{x}, t)=\frac{1}{4 \pi t \kappa} \exp \left(-\frac{|\vec{x}|^{2}}{4 t \kappa}\right)
$$

satisfying

$$
\begin{cases}\frac{\partial \Phi(\vec{x}, t)}{\partial t}-\kappa \nabla^{2} \Phi=0 & \text { in } \mathbb{R}^{2} \times(0, \infty) \\ \Phi(\vec{x}, 0)=\delta_{0} & \text { on } \mathbb{R}^{2} \times\{t=0\} .\end{cases}
$$

Then convolution of the forcing function and the Green's function $\hat{T}(\vec{x}, t ; s)=\Phi(\vec{x}, t-s) *$ $f(\vec{x}, s)$ satisfies the parameterized initial value problem

$$
\begin{cases}\frac{\partial \hat{T}(\vec{x}, t ; s)}{\partial t}-\kappa \nabla^{2} \hat{T}=0 & \text { in } \mathbb{R}^{2} \times(0, \infty) \\ \hat{T}(\vec{x}, t ; s)=f(\vec{x}, s) & \text { on } \mathbb{R}^{2} \times\{t=0\} .\end{cases}
$$

Duhamel's Principle states that the forcing function can be viewed as a continuous application of impulses [15], so that $T(\vec{x}, t)=\int_{0}^{t} \hat{T}(\vec{x}, t ; s) d s+T_{0}$ satisfies the given PDE. Thus

$$
\begin{aligned}
T(\vec{x}, t) & =\int_{0}^{t} \hat{T}(\vec{x}, t ; s) d s+T_{0} \\
& =\frac{2 P}{\pi k} \int_{0}^{t} \frac{\exp \left(-\frac{2\left|\vec{x}-\vec{x}_{l}\right|^{2}}{\omega^{2}+8 \kappa(t-s)}\right)}{\frac{\omega^{2}+8 \kappa(t-s)}{\kappa}} d s+T_{0} \\
& =\frac{P}{4 \pi k} \int_{\frac{2 r^{2}}{8 \kappa t+\omega^{2}}}^{\frac{2 r^{2}}{\omega^{2}}} \frac{\exp (-s)}{s} d s+T_{0} \\
& =\frac{P}{4 \pi k}\left(\operatorname{Ei}\left(-\frac{2 r^{2}}{\omega^{2}}\right)-\operatorname{Ei}\left(-\frac{2 r^{2}}{\omega^{2}+8 \kappa t}\right)\right)+T_{0},
\end{aligned}
$$

where $\operatorname{Ei}(z)=-\int_{-z}^{\infty} \frac{\exp (-t)}{t} d t$ and $r=\left|\vec{x}-\vec{x}_{l}\right|$.

To visualize this function, we use a dimensional reduction method to form a scaled temperature response [23]. Within this, we aggregate all of the material and laser parameters into a single distance-like, and a single time-like variable, so that the general temperature response can be plotted in three dimensions. We first find the proper temperature scaling factor. Let $T_{c}(t)=\lim _{r \rightarrow 0} T(r, t)$. Then

$$
\begin{aligned}
T_{c} & =\lim _{r \rightarrow 0}\left[\frac{P}{4 \pi k}\left(\operatorname{Ei}\left(-\frac{2 r^{2}}{\omega^{2}}\right)-\operatorname{Ei}\left(-\frac{2 r^{2}}{\omega^{2}+8 \kappa t}\right)\right)+T_{0}\right] \\
& =\frac{P}{4 \pi k} \ln \left(\frac{8 \kappa t}{\omega^{2}}+1\right)+T_{0} \\
& =A(t) \frac{P}{4 \pi k}+T_{0},
\end{aligned}
$$


and $\bar{T}=\left(T-T_{0}\right) / T_{c}$ is the scaled temperature function. Note also that this expression for $T_{c}$ affords a way to compute the peak temperature of the response, without evaluating an integral. Next, we define a dimensionless variable to represent the distance of a point to the laser peak position, $\mathcal{X}=2 r / \omega$, which scales linearly with $r$. Finally, we define $\mathcal{T}=\left(\omega^{2}+8 \kappa t\right) / \omega^{2}$, which scales linearly with $t$, inspired by the expression of $T(r, t)$. The nondimensionalized temperature response is then

$$
\bar{T}(\mathcal{X}, \mathcal{T})=A \int_{\mathcal{X}^{2} / \mathcal{T}}^{\mathcal{X}^{2}} \frac{e^{-z}}{z} d z
$$

Figure 12 shows a surface plot of this function for $A=1$. The temperature response for any particular system is found by calculating $A, \mathcal{X}$, and $\mathcal{T}$ from the desired parameters and sweeping $\bar{T}(\cdot, \mathcal{T})$ over a circle to make a radially symmetric response.

\section{Appendix B. MCMC Details}

The Markov chain Monte Carlo method is developed for the efficient application of Bayes' rule for conditional probability. Define $\vec{\theta}$ to be a vector of values for the parameters of interest (e.g. model parameters instantiating a given flaw context), $\vec{T}$ to be the actual temperature response at all measured spatio-temporal locations, and $\vec{T}^{*}=\vec{T}+\vec{\eta}$ to be the measured response under $\vec{\eta}$ assumed measurement noise. We seek the probability density function of the crack parameters given a particular noisy thermal measurement, $p\left(\vec{\theta} \mid \overrightarrow{T^{*}}\right)$, the posterior distribution. Bayes' rule states that this is proportional to the product of $p\left(\vec{T}^{*} \mid \vec{\theta}\right)$ (the likelihood distribution of a measured response given a particular set of crack parameters) and $p(\vec{\theta})$ (the prior distribution which contains all the beliefs regarding plausible crack parameters).

In our case, we make minimal assumptions regarding the crack elliptical geometry, and the prior distribution is uniform over a wide range of values for each subsequent parameter (i.e. their support). Our measured response is taken over a $3 \times 3$ pixel patch, within which the crack is assumed to be located; thus the prior distributions for the horizontal and vertical location of the crack center are uniform over this area, while the prior distribution of the crack length is taken to be uniform from zero to the length of one pixel. We also assume that the additive noise $\vec{\eta}$ over each pixel is i.i.d. Gaussian, with a standard deviation equal to the NETD of the considered imaging system, $p\left(\eta_{i}\right)=(2 \pi \sigma)^{-1 / 2} \exp \left(-\eta_{i}^{2} /(2 \sigma)\right)$. This PDF is to be used in forming the likelihood distribution.

We wish to be able to evaluate the likelihood function $p\left(\vec{T}^{*} \mid \vec{\theta}\right)$ for many values of $\vec{\theta}$. To do this, we use the finite element modeling software to simulate a temperature response over a specified crack instance, $\vec{T}(\vec{\theta})$ and substitute this response into the likelihood function (i.e. the likelihood is closed within a computer model). Then the probability that our noisy thermal measurement was caused by this crack is governed by the probability that each pixel reading had realized the difference between its measured value and the simulated response 
as measurement noise

$$
\begin{aligned}
p\left(\vec{T}^{*} \mid \vec{\theta}\right) & =p_{\eta}\left(T_{1}(\vec{\theta})-T_{1}^{*}, \ldots, T_{9}(\vec{\theta})-T_{9}^{*}\right) \\
& =\prod_{i} p_{\eta}\left(T_{i}(\vec{\theta})-T_{i}^{*}\right) \\
& =\prod_{i} \frac{1}{\sqrt{2 \pi \sigma}} \exp \left(-\frac{\left(T_{i}(\vec{\theta})-T_{i}^{*}\right)^{2}}{2 \sigma}\right) \\
& =\frac{1}{\sqrt{2 \pi \sigma}} \exp \left(-\frac{\sum_{i}\left(T_{i}(\vec{\theta})-T_{i}^{*}\right)^{2}}{2 \sigma}\right) .
\end{aligned}
$$

We have now defined the necessary components to perform MCMC sampling of the posterior distribution. At each step $k$ in the chain, we have evaluated the likelihood $p^{(k)}=p\left(\vec{T}^{*} \mid \vec{\theta}^{(k)}\right)$. A candidate $\vec{\theta}^{*}$ is chosen randomly according to a multivariate uniform distribution with width $2 \vec{L}$ centered at $\vec{\theta}^{(k)}$, and the candidate likelihood $p^{*}=p\left(\vec{T}^{*} \mid \vec{\theta}^{*}\right)$ is computed. The ratio of these two likelihoods determines how the chain moves. If the new location $\vec{\theta}^{*}$ has higher likelihood than the previous location, then it is selected as $\vec{\theta}^{(k+1)}$. Otherwise, the chain moves to the new location with probability $p^{*} / p^{(k)}$ and remains put, $\vec{\theta}^{(k+1)}=\vec{\theta}^{(k)}$ with probability $1-p^{*} / p^{(k)}$. This permission to move to a less likely parameter is the key to the Metropolis-Hastings algorithm. Under our condition that the chain can transition from any state $\vec{\theta}$ to any other state $\vec{\theta}^{\prime}$ in a finite number of steps (irreducibility of the chain), the MCMC samples are guaranteed to converge to a unique, stationary posterior distribution $[20]$.

In our inverse problem solution, we simultaneously solve for three crack parameters encoded in $\vec{\theta}$. In practice, each parameter is updated one-at-a-time, so every step of the chain requires three evaluations of $p^{(k)}$, with one updated parameter each time. This process is known as Metropolis-within-Gibbs sampling. The estimated PDF of each crack parameter is simply the marginal distribution of the MCMC samples.

The convergence of MCMC samples occurs at a faster rate if subsequent samples have low correlation. Typically, a burn-in period is used to ensure independence of the sample from the initial location. We also use the burn-in period to tune the width of the candidate distribution for each parameter, $L$ [24]. A narrow candidate distribution will result in relatively high probability of accepting the new candidate at each step, but the candidates will be close to the previous samples. On the other hand, a wide candidate distribution can provide distant candidates, but a lower acceptance probability implies that many subsequent samples will be identical. Balancing these factors motivates the tuning of $L$ for each chain during burn-in. By increasing $L$ by a small factor after each accepted burn-in sample and decreasing it by some related factor after each rejection, it can be tuned to find a value associated with a certain probability of acceptance. The literature suggests a target acceptance probability of 0.42 [24], but this was found to produce samples with very high correlation in our three parameter chains. We performed several test chains with fixed values of $L$ for the uncracked problem to determine the proper acceptance probability for 
our problem. Figure 13 shows empirical lag 1 autocorrelations and acceptance probabilities for varying values of $L$. It is determined that an acceptance probability near 0.1 is associated with minimal autocorrelation for all three crack parameters; thus this value was used as the target for the burn-in periods of all MCMC simulations presented in this work.

\section{Appendix C. Convergence Analysis}

In order to be confident that our MCMC samplings are sufficiently long to provide good estimates of the posterior distribution $p\left(\vec{\theta} \mid \vec{T}^{*}\right)$, we carry out a separate, independent convergence study. We analyze here two sets of MCMC samplings, each having a single noise realization across $m=10$ trials. The $m$ chains are initialized randomly across the support of the prior distribution, with each sampled uniformly from a disjoint interval spanning one $m^{\text {th }}$ of the width of the prior. This is so that we have confidence that the MCMC samples that we collect after the burn-in period are valid from any random initial point. Each chain has 5,000 discarded burn-in samples and 10,000 saved samples.

The convergence diagnostic we use is based on $(1-\alpha)$ credible intervals [25]. From each individual chain, the empirical $(1-\alpha)$ credible intervals are computed. For each withinchain interval, the proportion of samples from all $m$ chains that occur within this interval is determined. Finally, these proportions are averaged to give the convergence diagnostic. If all $m$ chains have converged to the same distribution, then the convergence diagnostic will converge to $(1-\alpha)$. Figure 14 shows trajectories of this value as the first set of simulations evolves with $\alpha=0.05$. The convergence diagnostic is seen to converge to $(1-\alpha)$ within the 10,000 samples used. The behavior is the same with the second set of simulations and for any value of $\alpha$. We are therefore confident that the simulations presented in Section 4.4, which have 20,000 samples, are reliable estimates of the desired distribution.

\section{References}

[1] T. Li, D. P. Almond, D. S. Rees, Crack imaging by scanning laser-line thermography and laserspot thermography, Measurement Science and Tecnhonolgy 22 (3) (2011) 035701. doi:10.1088/09570233/22/3/035701.

[2] T. Li, D. P. Almond, D. S. Rees, Crack imaging by scanning pulsed laser spot thermography, NDT\&E International 44 (2) (2011) 216-225. doi:10.1016/j.ndteint.2010.08.006.

[3] J. Schlichting, C. Maierhofer, M. Kreutzbruck, Crack sizing by laser excited thermography, NDT\&E International 45 (1) (2012) 133-140. doi:10.1016/j.ndteint.2011.09.014.

[4] S. Burrows, S. Dixon, S. Pickering, T. Li, D. Almond, Thermographic detection of surface breaking defects using a scanning laser source, NDT\&E International 44 (7) (2011) 589-596. doi:10.1016/j.ndteint.2011.06.001.

[5] K. Chatterjee, S. Tuli, S. G. Pickering, D. P. Almond, A comparison of the pulsed, lock-in and frequency modulated thermography nondestructive evaluation techniques, NDT\&E International 44 (7) (2011) 655-667. doi:10.1016/j.ndteint.2011.06.008.

[6] W. Jeong, C. Earls, W. Philpot, A. Zehnder, Inverse thermographic characterization of optically unresolvable through cracks in thin metal plates, Mechanical Systems and Signal Processing 27 (1) (2012) 634-650. doi:10.1016/j.ymssp.2011.09.021.

[7] W. N. Reynolds, Thermographic methods applied to industrial materials, Canadian Journal of Physics 64 (9) (1986) 1150-1154. doi:10.1139/cjp-2013-0128. 
[8] V. Vavilov, D. Burleigh, Pulsed thermal ndt in tables, figures and formulas, Thermosense: Thermal Infrared Applications XXXVII, 2015, pp. 1-15. doi:10.1117/12.2181039.

[9] J. Varis, Detection of vertical cracks in carbon fiber composites using an infrared line scanner, Springer US, 1993, Ch. 5, pp. 1223-1227. doi:10.1007/978-1-4615-5339-7_158.

[10] J. Krapez, C. Gruss, R. Huttner, F. Lepoutre, L. Legrandjacques, La caméra photothermique - partie i : Principe, modélisation, application à la détection de fissures, Instrumentation, Mesure, Métrologie 1 (1) (2001) 9-40.

[11] J. Krapez, F. Lepoutre, R. Huttner, C. Gruss, L. Legrandjacques, M. Piriou, J. Gros, D. Gente, S. Hermosilla-Lara, P. Joubert, D. Placko, La caméra photothermique - partie ii : Applications industrielles, perspectives damélioration par un nouveau traitement dimage, Instrumentation, Mesure, Métrologie 1 (1) (2001) 41-67.

[12] A. Rashed, D. Almond, D. Rees, S. Burrows, S. Dixon, Crack detection by laser spot imaging thermography, Review of Quantitative Nondestructive Evaluation, 2007, pp. 500-506. doi:10.1063/1.2718013.

[13] C. Earls, Stochastic inverse thermographic characterization of sub-pixel sized through cracks, Mechanical Systems and Signal Processing 30 (1) (2012) 146-156. doi:10.1016/j.ymssp.2012.01.016.

[14] K. Bryan, Efficient computational methods for thermal imaging of small cracks in plates, Present at the 2012 SIAM Annual Meeting, 2012.

[15] L. Evans, Partial Differential Equations, American Mathematical Society, 2010.

[16] J. Alda, Laser and gaussian beam propagation and transformation, Encyclopedia of optical engineering (2002) 999-1013.

[17] M. Kaviany, Principles of Heat Transfer, John Wiley \& Sons, Inc., 2002.

[18] S. Marinetti, V. Vavilov, Ir thermographic detection and characterization of hidden corrosion in metals: General analysis, Corrosion Science 52 (3) (2010) 865-872. doi:10.1016/j.corsci.2009.11.005.

[19] A. Logg, K.-A. Mardal, G. N. W. et al., Automated Solution of Differential Equations by the Finite Element Method, Springer, 2012. doi:10.1007/978-3-642-23099-8.

[20] W. Hastings, Monte carlo sampling methods using markov chains and their applications, Biometrika 57 (1) (1969) 97-109. doi:10.1093/biomet/57.1.97.

[21] J. F. Ready, Effects of High-Power Laser Radiation, Academic Press, 2012.

[22] M. Lax, Temperature rise induced by a laser beam, Journal of Applied Physics 48 (9) (1977) 3660. doi: $10.1063 / 1.324265$.

[23] M. H. Holmes, Introduction to the Foundations of Applied Mathematics, Vol. 56 of Texts in Applied Mathematics, Springer New York, 2009. doi:10.1007/978-0-387-87765-5.

[24] W. A. Link, R. J. Barker, Bayesian Inference with ecological applications, Academic Press, 2010.

[25] S. P. Brooks, A. Gelman, General methods for monitoring convergence of iterative simulations, Journal of Computational and Graphical Statistics 7 (4) (1998) 434-455. doi:10.1080/10618600.1998.10474787. 
Figure C.1: (left) Inspection schematic annotated with relevant domains for the mathematical idealization Equation (1). (right) Close-up view of the local flaw neighborhood with overlaid pixel boundaries from the IR imaging system. The crack location (center), major axis, and minor axis are marked.

Figure C.2: (top) 2D cross section of the radially symmetric analytical solution Equation (2) and (bottom) its gradient magnitude at varying durations of the laser pulse.

Figure C.3: Infinite array of images of a square domain with an example of the symmetric positions for a laser peak in each image.

Figure C.4: 2D cross sections of analytical solutions at varying distances to domain boundary, marked on the $x$ axis, with and without the use of images to satisfy the insulated boundary condition.

Figure C.5: 2D cross section of simulation response with varying laser peak positions, $\vec{x}_{l}=(0.22,0),(1.57,0)$, and $(6.5,0)$ for a crack location $\vec{x}_{c}=(0,0)$ with major axis in the $y$ direction, showing a larger jump in temperature when the laser is positioned so that $\vec{x}_{c}$ coincides with the location of maximal gradient of the temperature response.

Figure C.6: (left) Likelihood PDF of additive noise $\eta$ for various ratios of NETD/ $\Delta \mathrm{T}$ with $\mathrm{NETD}=1$. (right) Standard deviation of such PDF asymptotically approaching 1.

Figure C.7: (left) 2D cross section of the gradient magnitude function emphasized at seven laser offset distances. (right) Scatter plot of average MCMC posterior variance vs. analytical gradient for samplings performed using these distances.

Figure C.8: Maximal gradient magnitude as a function of pulse duration for an optimally-located laser spot as an example of asymptotic behavior. The dashed line denotes the limiting value.

Figure C.9: Vector diagram showing optimal laser location given a crack location over the $100 \mathrm{~mm} \times 100$ $\mathrm{mm}$ domain. The arrow bases are independently considered crack locations, with each arrow pointing at the optimal location for the laser peak to characterize a crack at its base. A circle denotes that a crack in its center is optimally characterized using a laser peak anywhere along its circumference: that is, asymmetry in the thermal gradient is negligible.

Figure C.10: (upper) Marginal posterior histograms for one of the twenty MCMC samplings in set 1, and (lower) set 2. Solid vertical lines denote the means of the samples, while dashed lines denote the true values used to produce surrogate inspection data.

Figure C.11: Joint posterior samples gathered from MCMC samplings used in the convergence study.

Figure C.12: Surface plot of the nondimensionalized response as a function of $\mathcal{X}$ and $\mathcal{T}$.

Figure C.13: (left) Experimental lag 1autocorrelation as a function of $L$ for the for uncracked problem. (right) Experimental acceptance probability of the same data. 
Figure C.14: Trajectories of the $(1-\alpha)$ convergence diagnostic for a representative pool of MCMC samplings. 

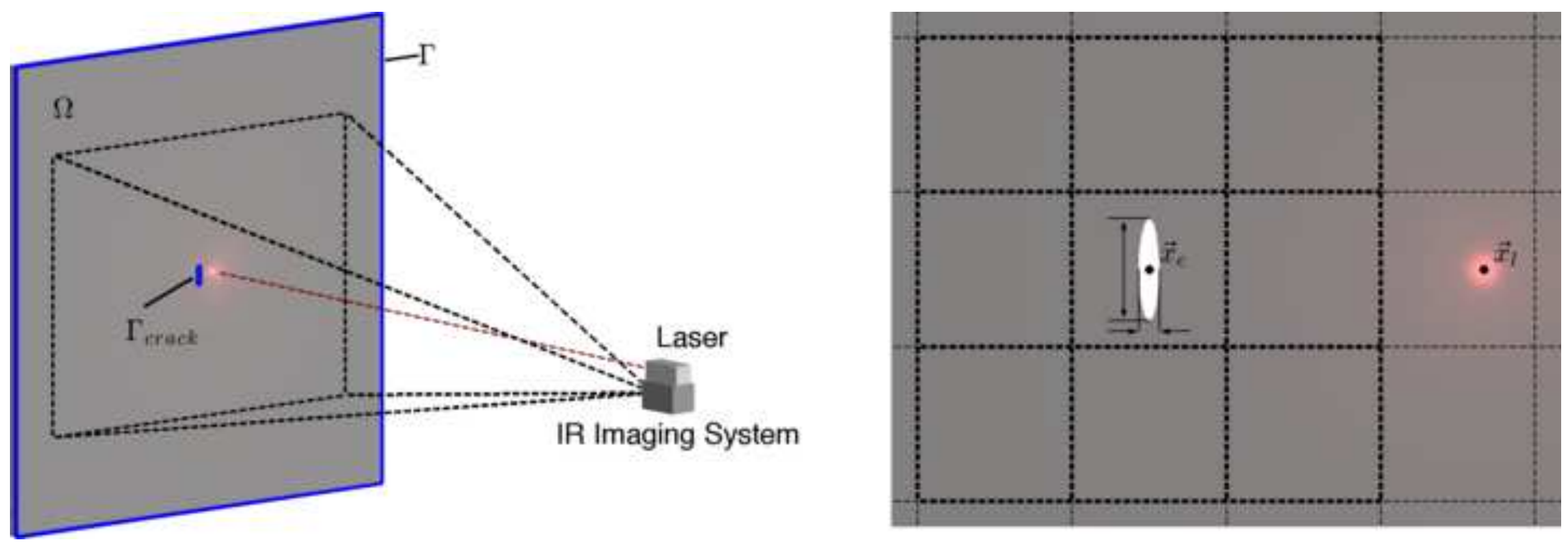


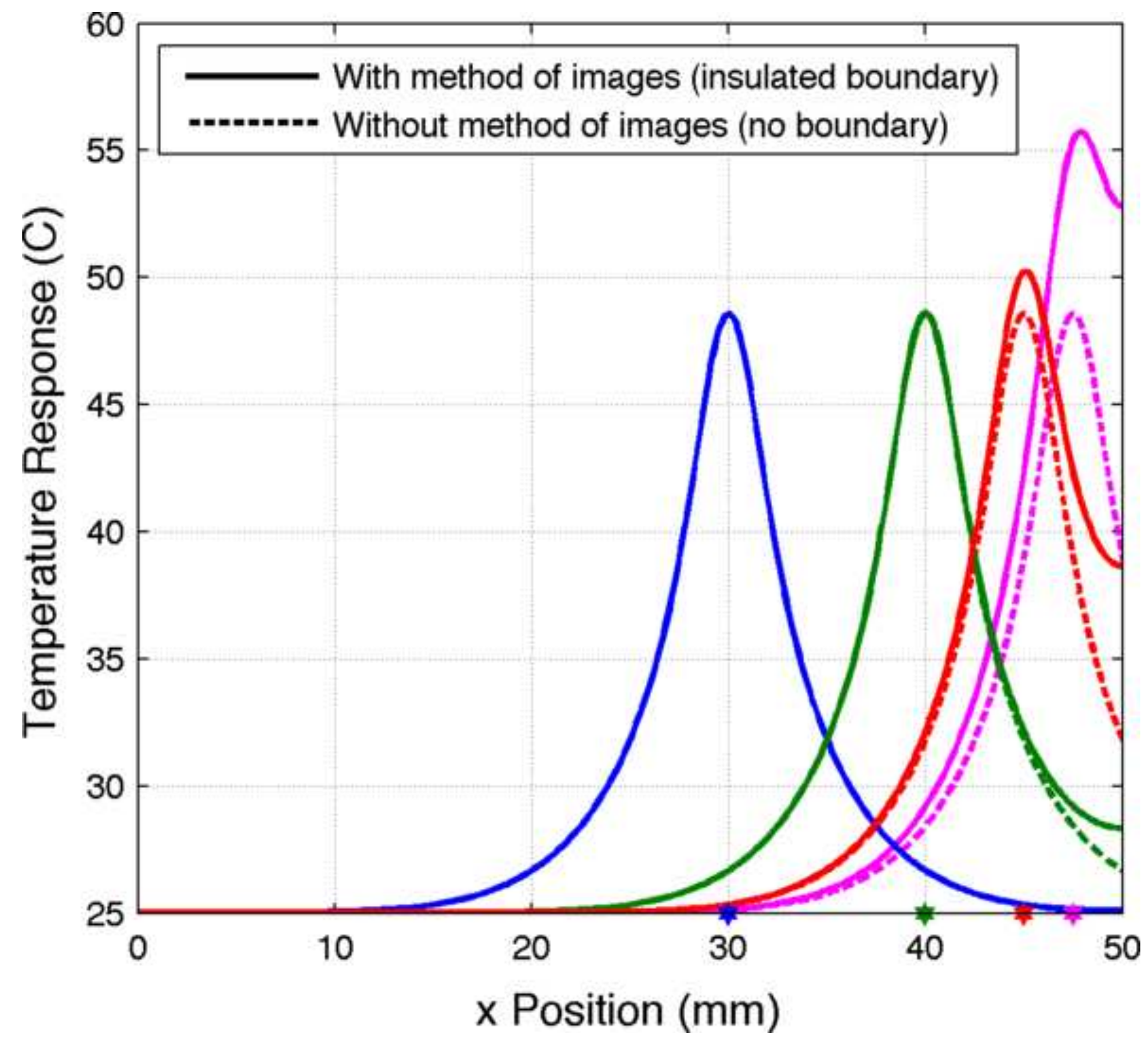




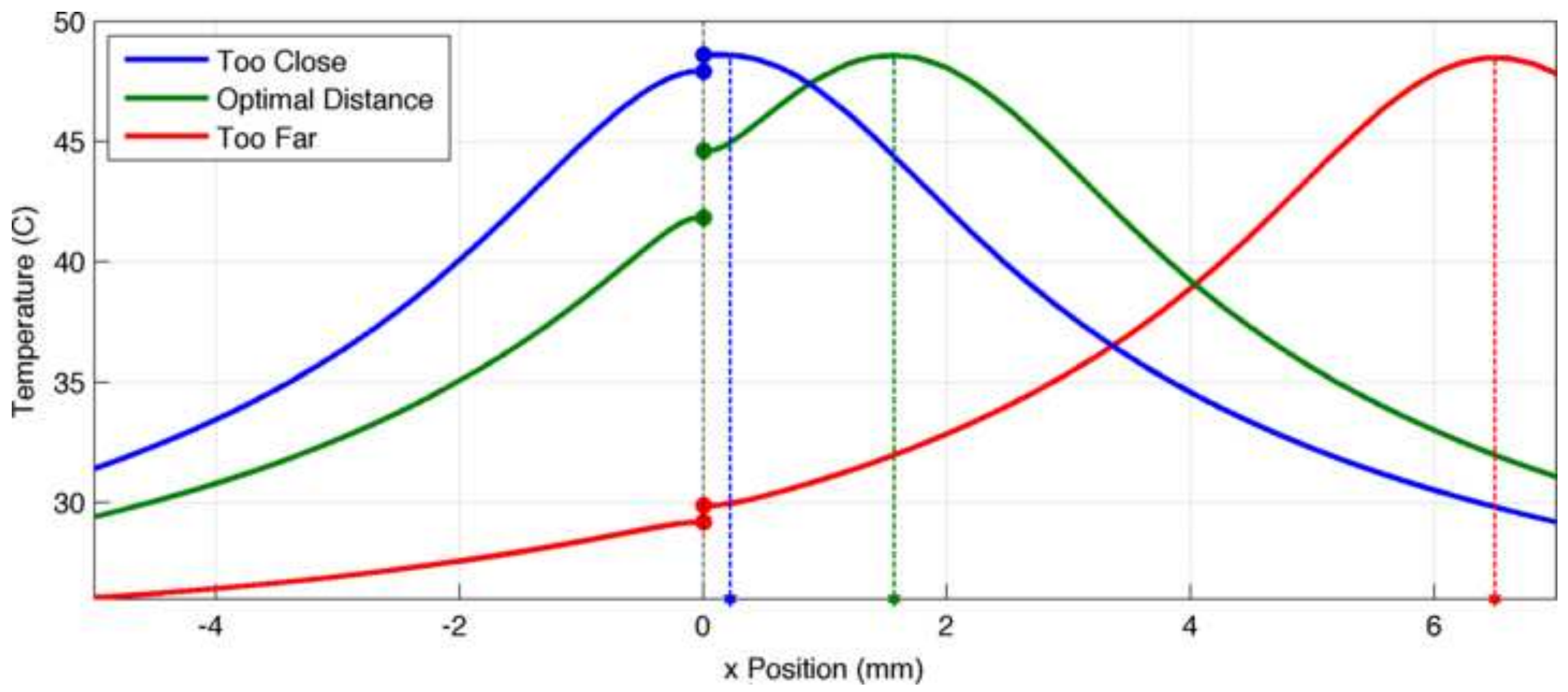



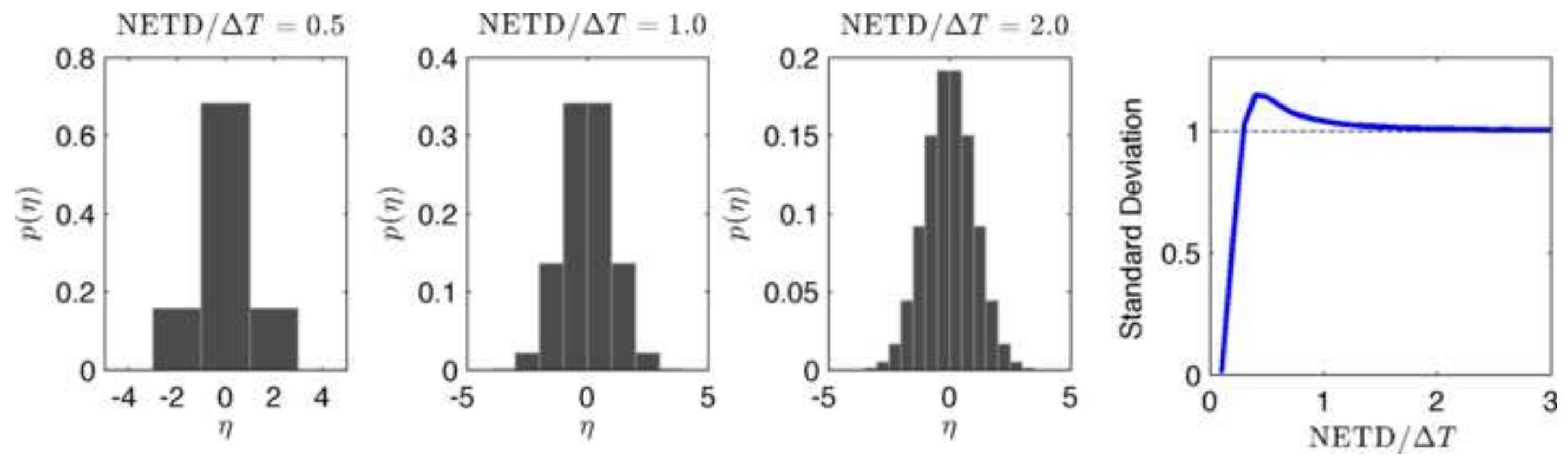

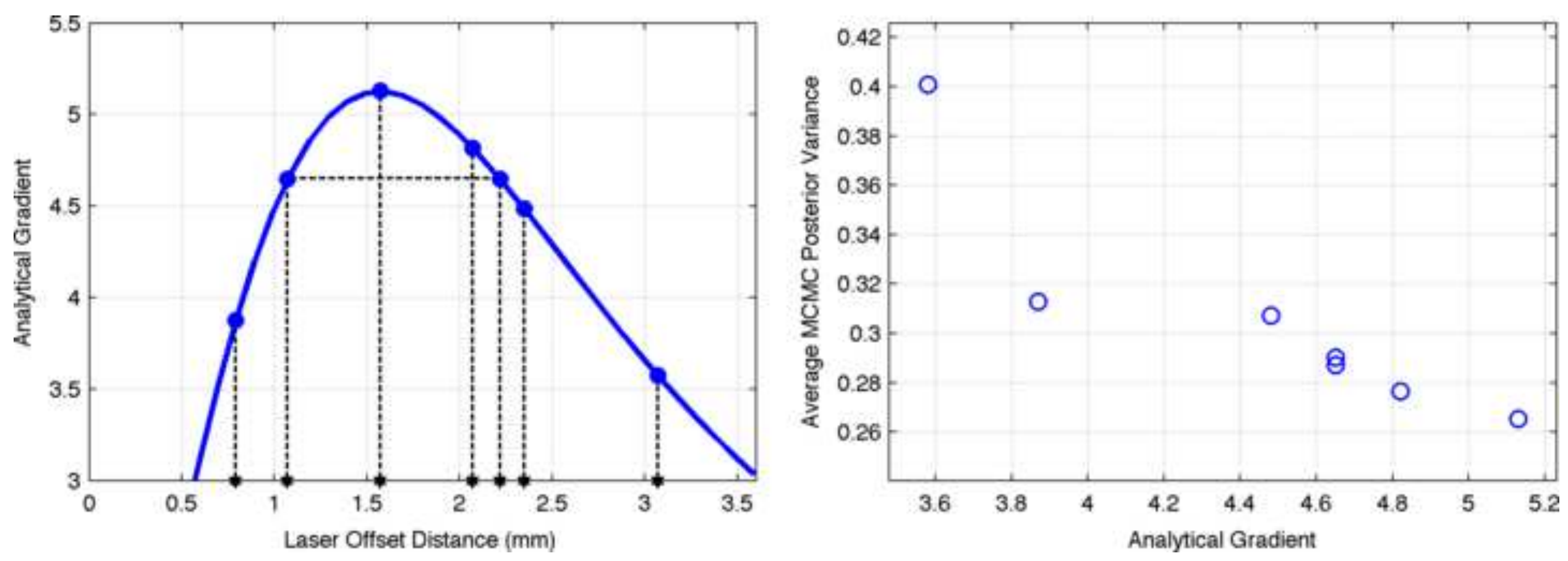


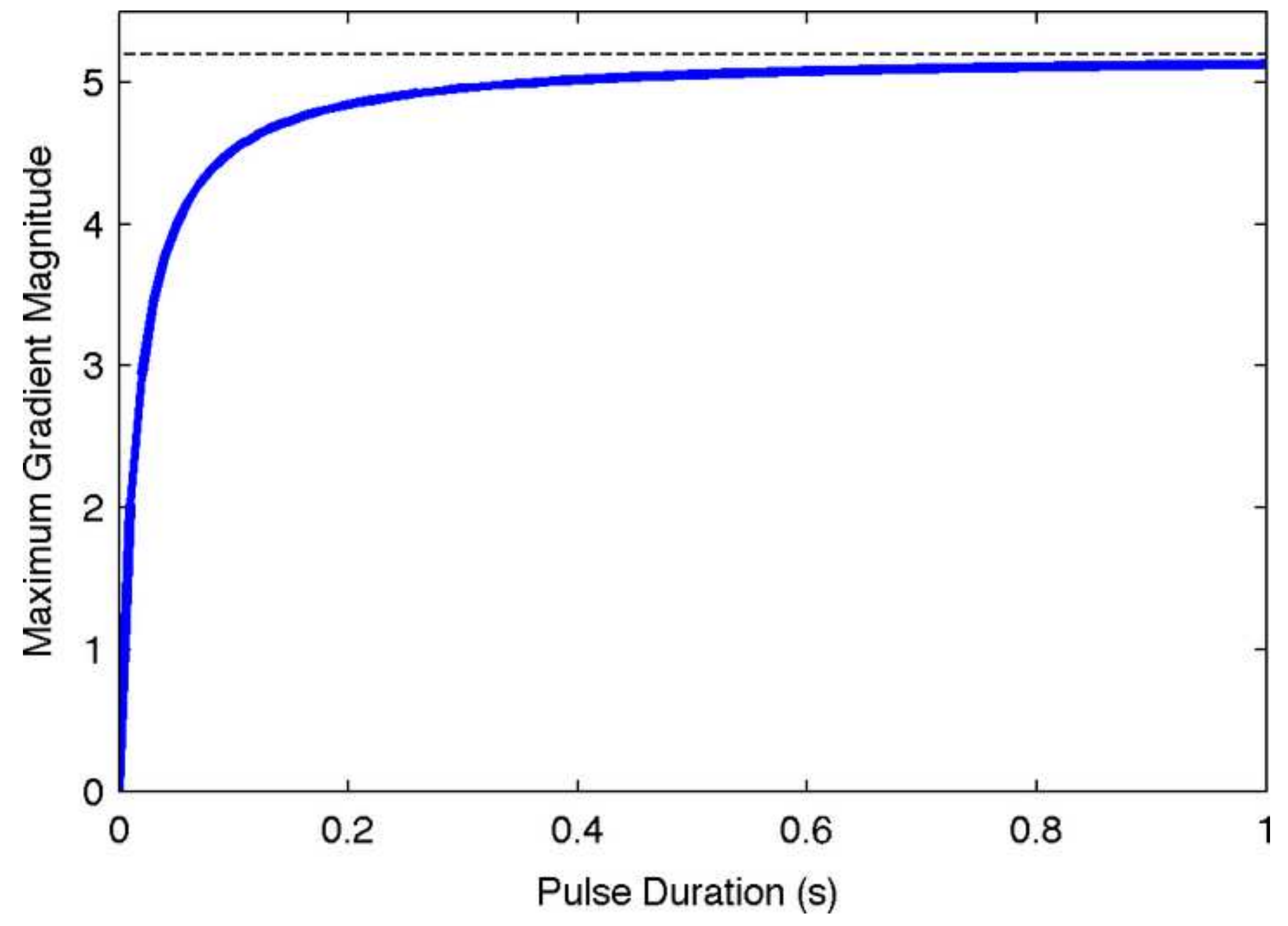




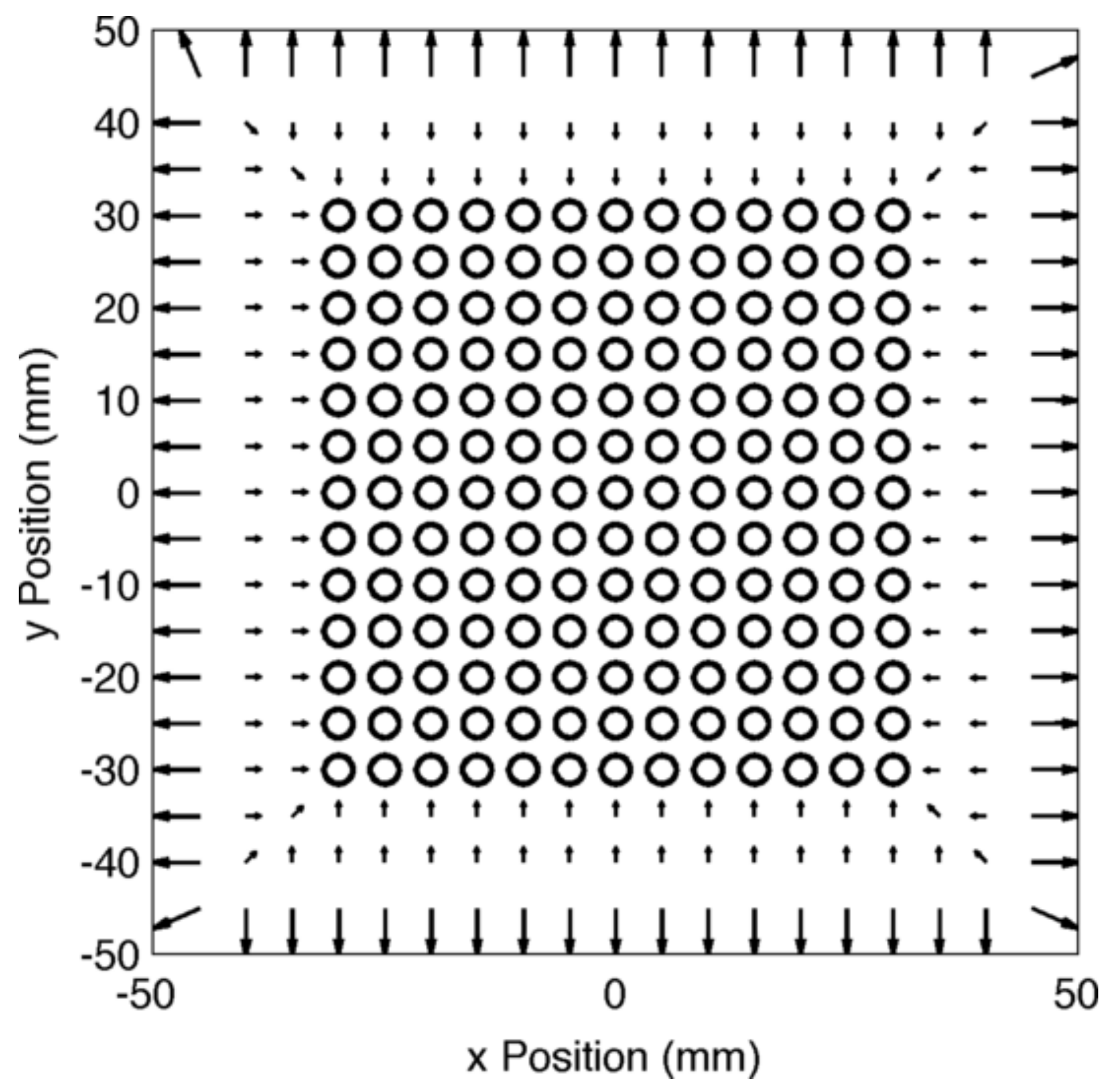



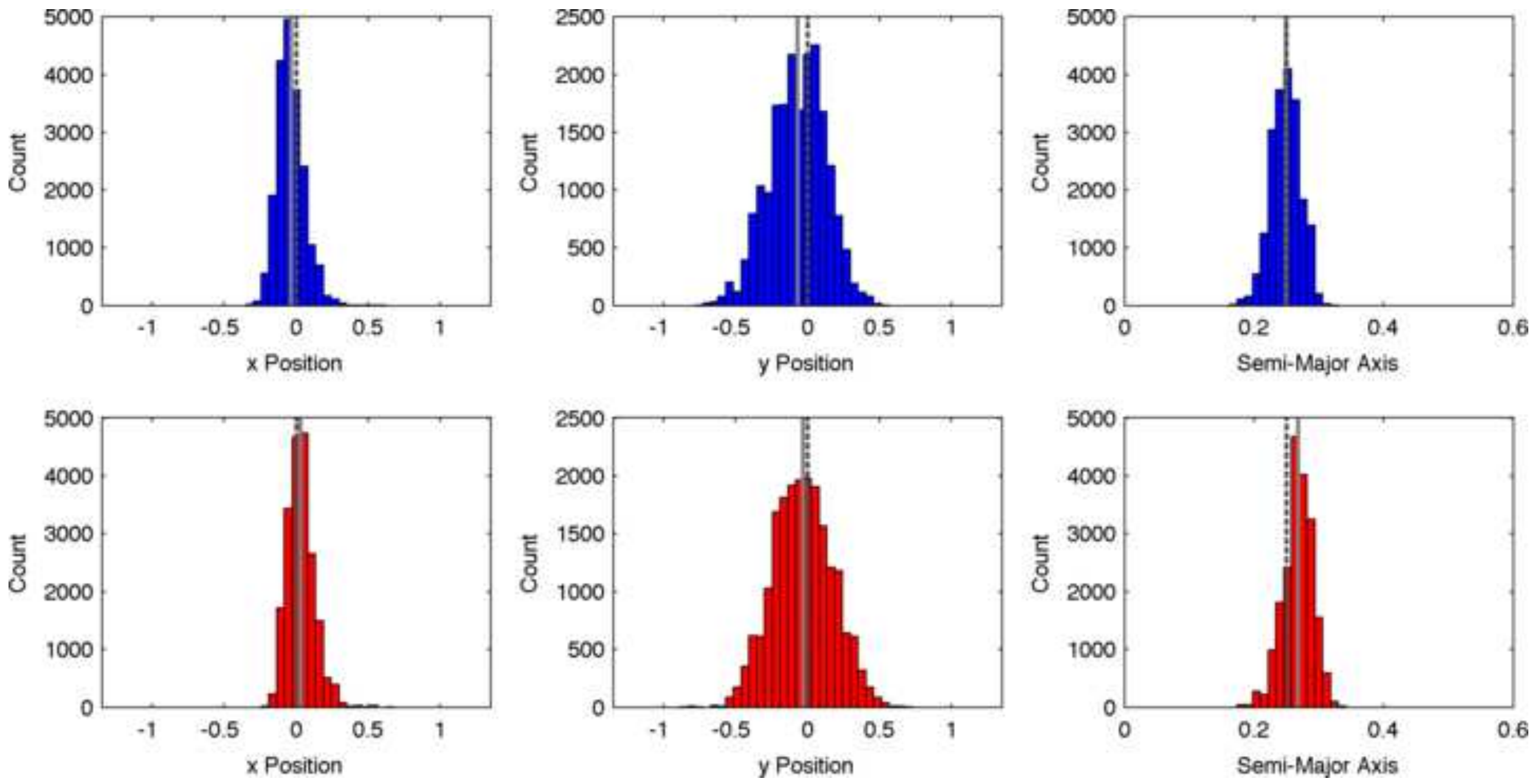

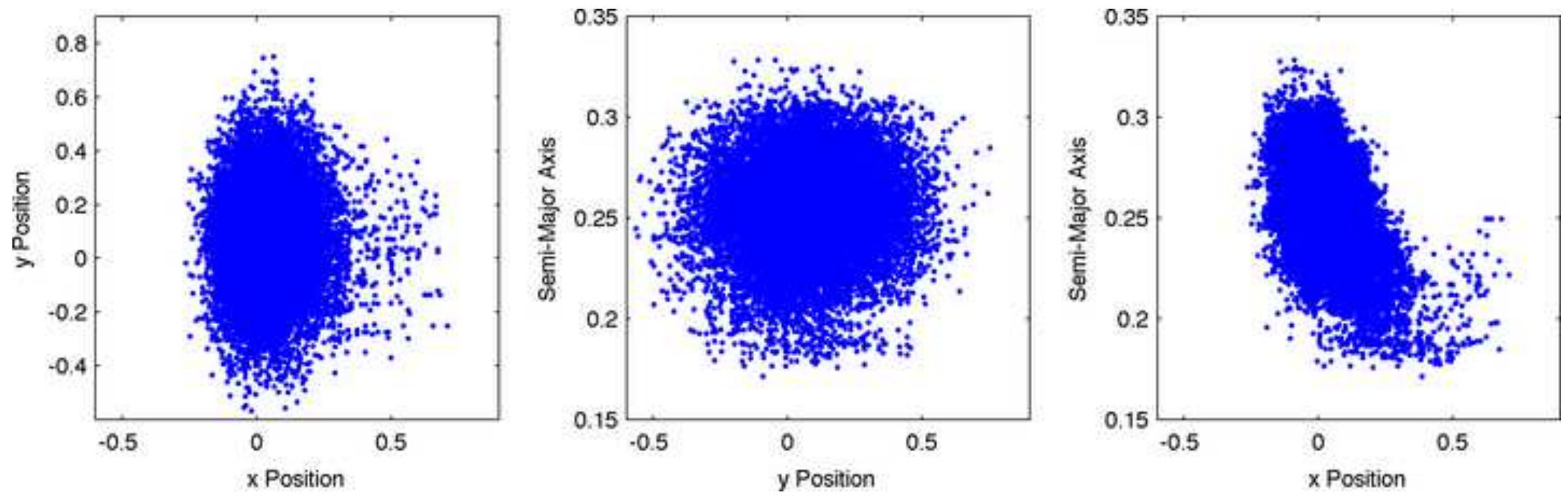


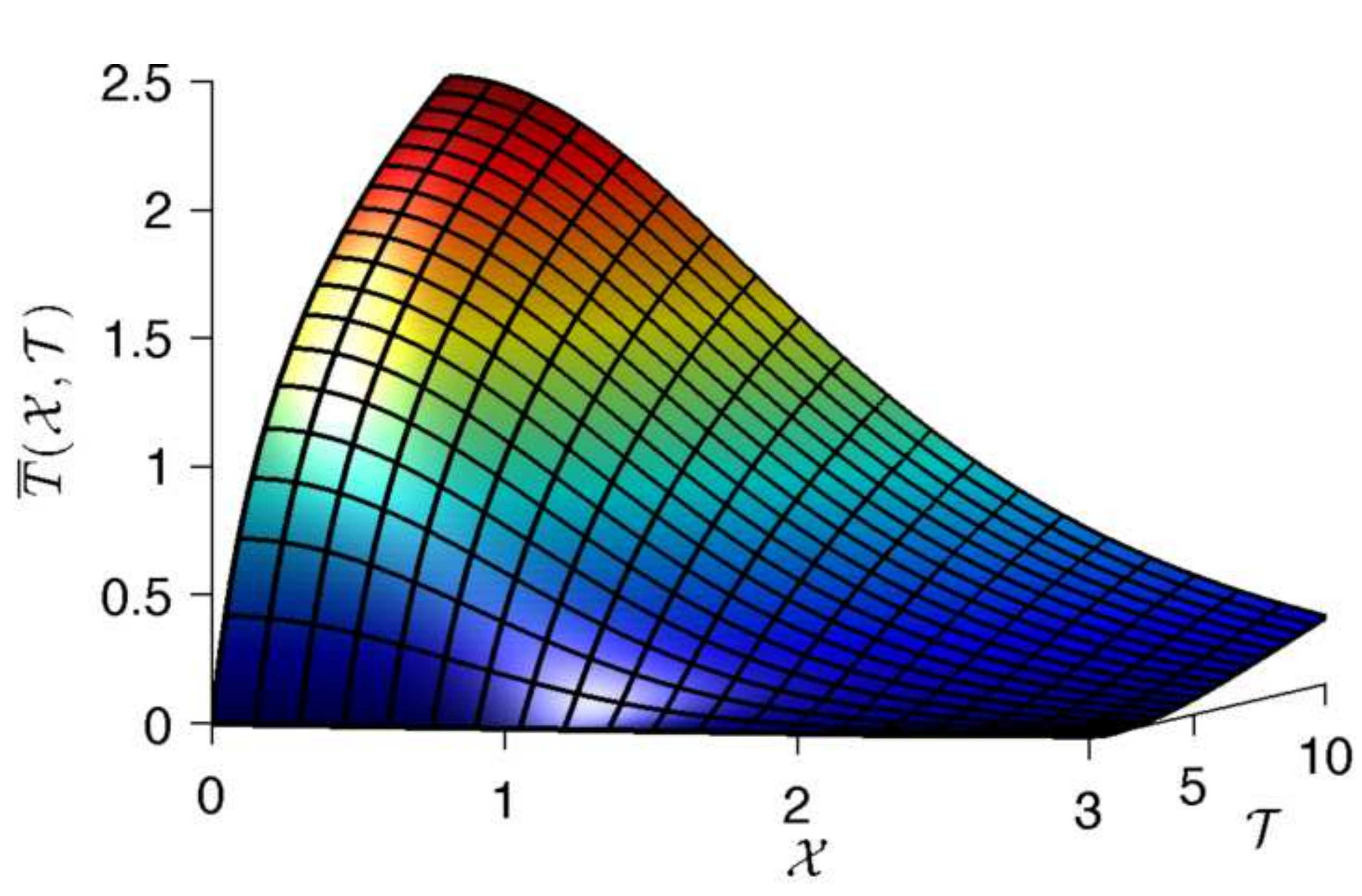

列

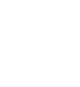

.

\begin{tabular}{l} 
de \\
d \\
\hline
\end{tabular}

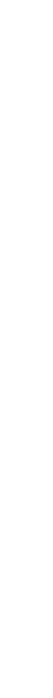

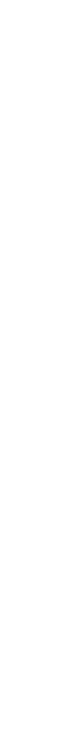



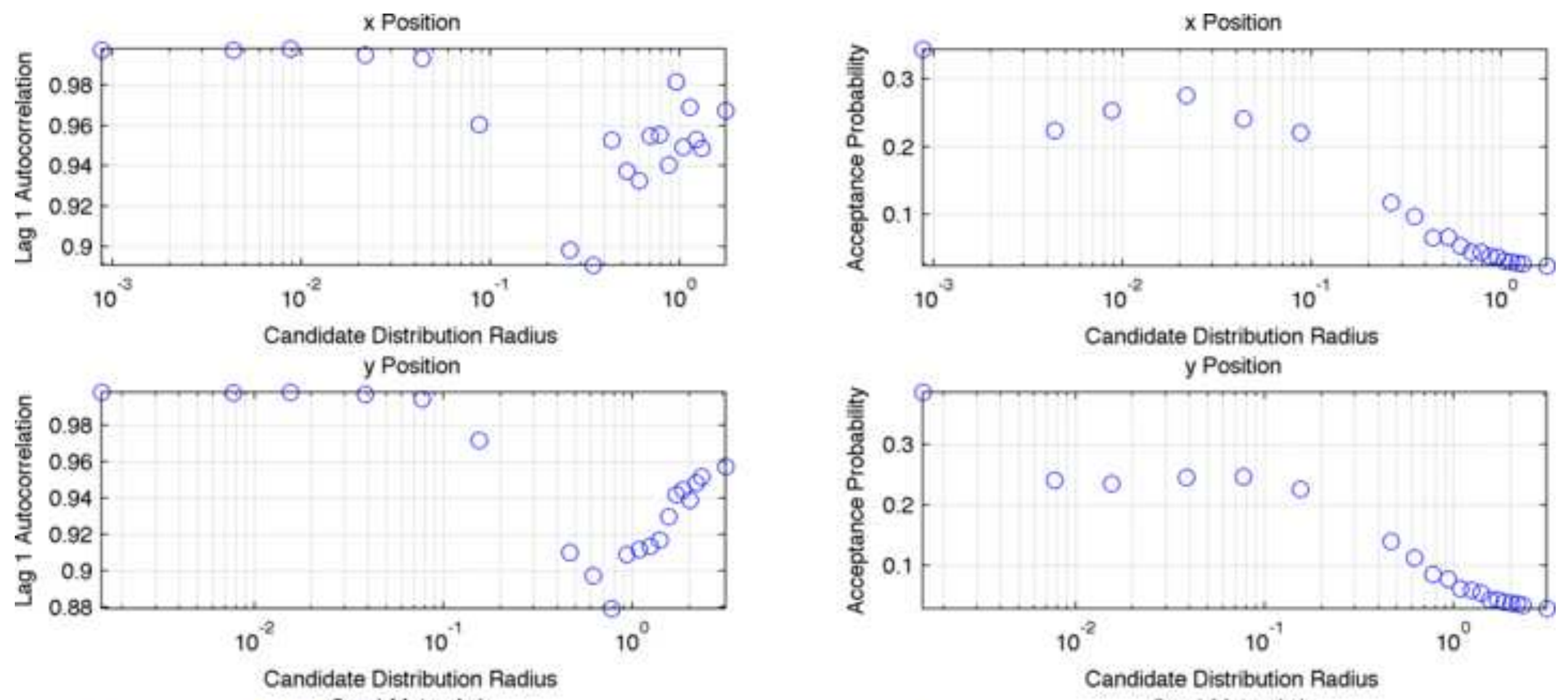

Semi-Major Axis
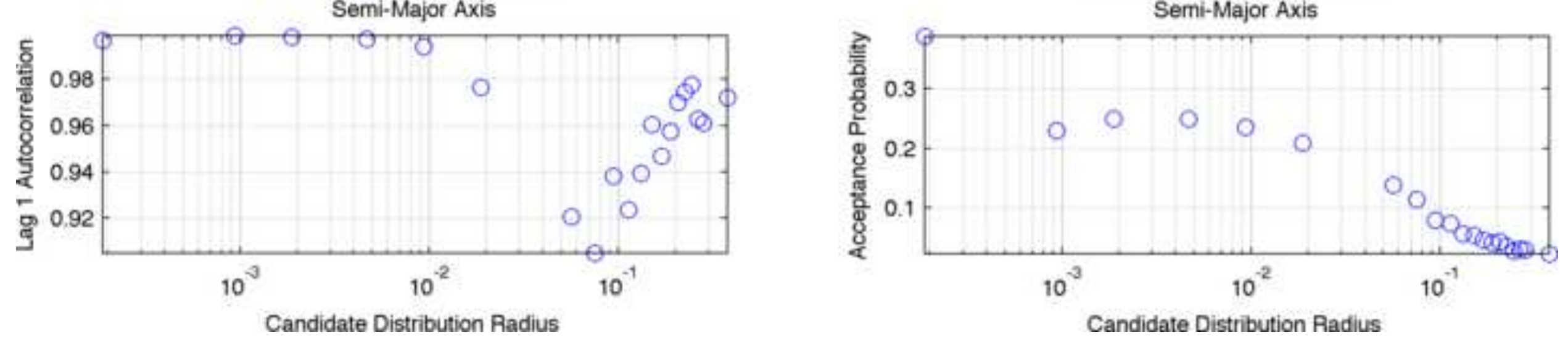

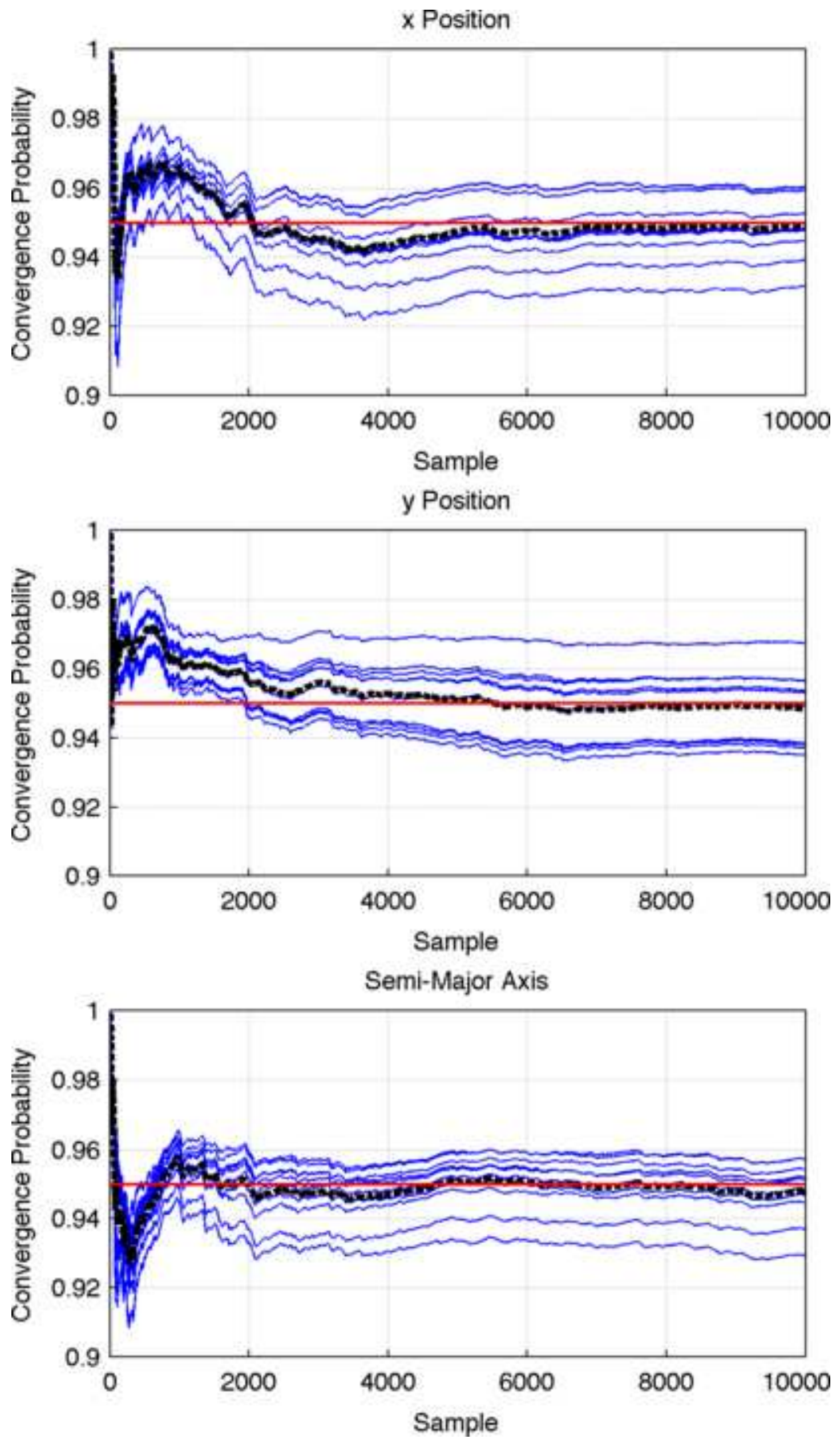